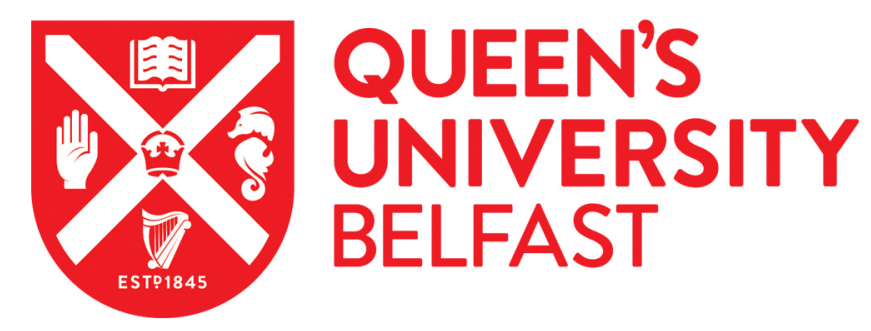

\title{
Future Internet: The Internet of Things Architecture, Possible Applications and Key Challenges
}

Khan, R., Khan, S. U., Zaheer, R., \& Khan, S. (2012). Future Internet: The Internet of Things Architecture, Possible Applications and Key Challenges. In 2012 10th International Conference on Frontiers of Information Technology (FIT): Proceedings (pp. 257-260). Institute of Electrical and Electronics Engineers Inc.. https://doi.org/10.1109/FIT.2012.53

Published in:

2012 10th International Conference on Frontiers of Information Technology (FIT): Proceedings

\section{Document Version:}

Peer reviewed version

Queen's University Belfast - Research Portal:

Link to publication record in Queen's University Belfast Research Portal

\begin{abstract}
Publisher rights
"๑ 2012 IEEE. Personal use of this material is permitted. Permission from IEEE must be obtained for all other uses, in any current or future media, including reprinting/republishing this material for advertising or promotional purposes, creating new collective works, for resale or redistribution to servers or lists, or reuse of any copyrighted component of this work in other works
\end{abstract}

\section{General rights}

Copyright for the publications made accessible via the Queen's University Belfast Research Portal is retained by the author(s) and / or other copyright owners and it is a condition of accessing these publications that users recognise and abide by the legal requirements associated with these rights.

Take down policy

The Research Portal is Queen's institutional repository that provides access to Queen's research output. Every effort has been made to ensure that content in the Research Portal does not infringe any person's rights, or applicable UK laws. If you discover content in the Research Portal that you believe breaches copyright or violates any law, please contact openaccess@qub.ac.uk. 


\title{
Future Internet: The Internet of Things Architecture, Possible Applications and Key Challenges
}

\author{
Rafiullah Khan ${ }^{*}$, Sarmad Ullah Khan ${ }^{\dagger}$, Rifaqat Zaheer ${ }^{\ddagger}$ and Shahid Khan ${ }^{\S}$ \\ * DITEN Dept. University of Genova (UNIGE), 16145 Genova, Italy, Email: rafiullah.khan@unige.it \\ $\dagger$ Electronics Dept. Politecnico Di Torino, 10129 Torino, Italy, Email: sarmad.khan@ polito.it \\ $\ddagger$ Electronics Dept. Politecnico Di Milano, 20133 Milano, Italy, Email: rifaqat.zaheer@mail.polimi.it \\ $\S$ Electrical Dept. COMSATS Institute of Information Technology, Abbottabad, Pakistan, Email: shahid@ciit.net.pk
}

\begin{abstract}
The Internet is continuously changing and evolving. The main communication form of present Internet is humanhuman. The Internet of Things (IoT) can be considered as the future evaluation of the Internet that realizes machine-to-machine (M2M) learning. Thus, IoT provides connectivity for everyone and everything. The IoT embeds some intelligence in Internetconnected objects to communicate, exchange information, take decisions, invoke actions and provide amazing services. This paper addresses the existing development trends, the generic architecture of IoT, its distinguishing features and possible future applications. This paper also forecast the key challenges associated with the development of IoT. The IoT is getting increasing popularity for academia, industry as well as government that has the potential to bring significant personal, professional and economic benefits.
\end{abstract}

Keywords-Internet of Things, ubiquitous computing, RFID, IoT architecture, IoT applications, IoT security.

\section{INTRODUCTION}

The Internet of Things (IoT) provides connectivity for anyone at any time and place to anything at any time and place. With the advancement in technology, we are moving towards a society, where everything and everyone will be connected [1]. The IoT is considered as the future evaluation of the Internet that realizes machine-to-machine (M2M) learning [2]. The basic idea of IoT is to allow autonomous and secure connection and exchange of data between real world devices and applications [3]. The IoT links real life and physical activities with the virtual world [4].

The numbers of Internet connected devices are increasing at the rapid rate. These devices include personal computers, laptops, tablets, smart phones, PDAs and other hand-held embedded devices. Most of the mobile devices embed different sensors and actuators that can sense, perform computation, take intelligent decisions and transmit useful collected information over the Internet [5]. Using a network of such devices with different sensors can give birth to enormous amazing applications and services that can bring significant personal, professional and economic benefits [6].

The IoT consists of objects, sensor devices, communication infrastructure, computational and processing unit that may be placed on cloud, decision making and action invoking system [7]. The objects have certain unique features and are uniquely identifiable and accessible to the Internet. These physical objects are equipped with Radio-Frequency IDentification (RFID) tags or other identification bar-codes that can be sensed by the smart sensor devices [6]. The sensors communicate object specific information over the Internet to the computational and processing unit. A combination of different sensors can be used for the design of smart services. The result of processing is then passed to the decision making and action invoking system that determines an automated action to be invoked.

This paper addresses the existing development trends, the generic architecture of IoT, IoT distinguishing features and possible future applications. The IoT is a hot research topic that is getting increasing popularity for academia, industry as well as government. Many European and American organizations and multinatinal companies are involved in the design and development of IoT to achieve different type of useful and powerful automated services [1]. The IoT has to face many challenges in its deployment specially in the field of security, governance and standardization that are also addressed in this paper.

The rest of the paper is organized as follows. Section II describes briefly the evolution of Internet. Section III presents the generic architecture of IoT. Section IV forecasts possible future application of IoT. Section V describes key challenges in the design and implementation of IoT. Finally, Section VI concludes the paper.

\section{Evaluation of InTERnet of Things}

The Internet has tremendously evolved in the last few years connecting billions of things globally. These things have different sizes, capabilities, processing and computational power and support different kind of applications [4]. Thus, the traditional Internet merges into smart future Internet, called IoT [1]. The generic scenario of IoT is shown in Fig. 1. The IoT connects real world objects and embeds the intelligence in the system to smartly process the object specific information and take useful autonomous decisions [2]. Thus, IoT can give birth to enormous useful applications and services that we never imagined before [1].

With the advancement in technology, the devices processing power and storage capabilities significantly increased while their sizes reduced. These smart devices are usually equipped with different type of sensors and actuators. Also these devices 


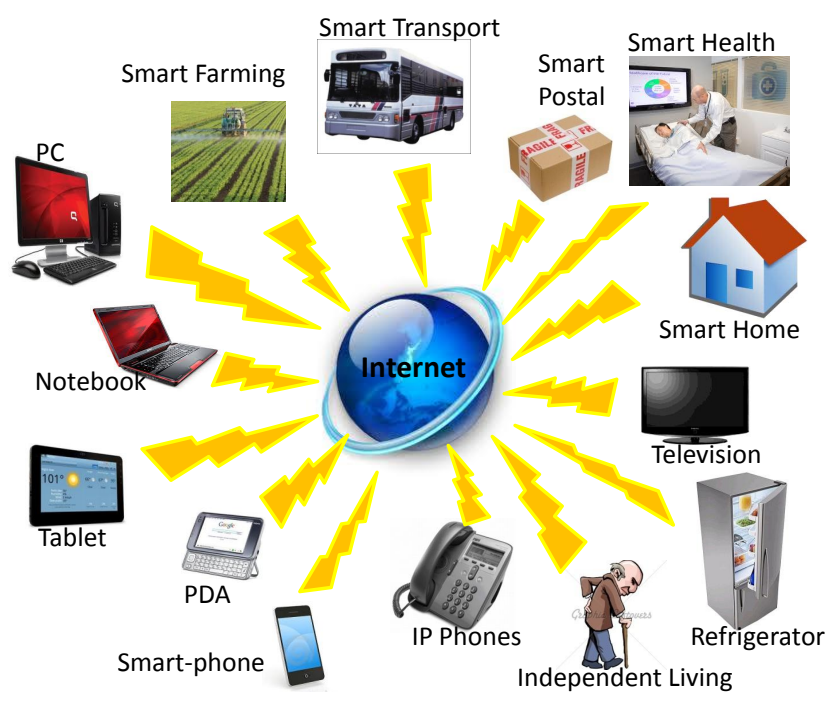

Figure 1: The IoT generic scenario.

are able to connect and communicate over the Internet that can enable a new range of opportunities [3]. Moreover, the physical objects are increasingly equipped with RFID tags or other electronic bar codes that can be scanned by the smart devices, e.g., smart phones or small embedded RFID scanner. The objects have unique identity and their specific information are embedded in the RFID tags. In 2005, the International Telecommunications Union (ITU) proposed that "Internet of Things" will connect the real world objects in both a sensory and intelligent manner [8]. Fig. 2 shows basic IoT system implementing different type of applications or services. The things connect and communicate with other things that implement the same service type. The basic simplified workflow of IoT can be described as follows:

1) Object sensing, identification and communication of object specific information. The information is the sensed data about temperature, orientation, motion, vibration, acceleration, humidity, chemical changes in the air etc depending on the type of sensors. A combination of different sensors can be used for the design of smart services.

2) Trigger an action. The received object information is processed by a smart device/system that then determines an automated action to be invoked.

3) The smart device/system provide rich services and includes a mechanism to provide feedback to the administrator about the current system status and the results of actions invoked.

\section{Generic Architecture}

Today's Internet is using TCP/IP protocol stack for communication between network hosts which was proposed long time ago. However, the IoT connects billions of objects which will create much larger traffic and much more data storage is needed [9]. Also, IoT will face many challenges specially related to privacy and security [10]. Thus, the new

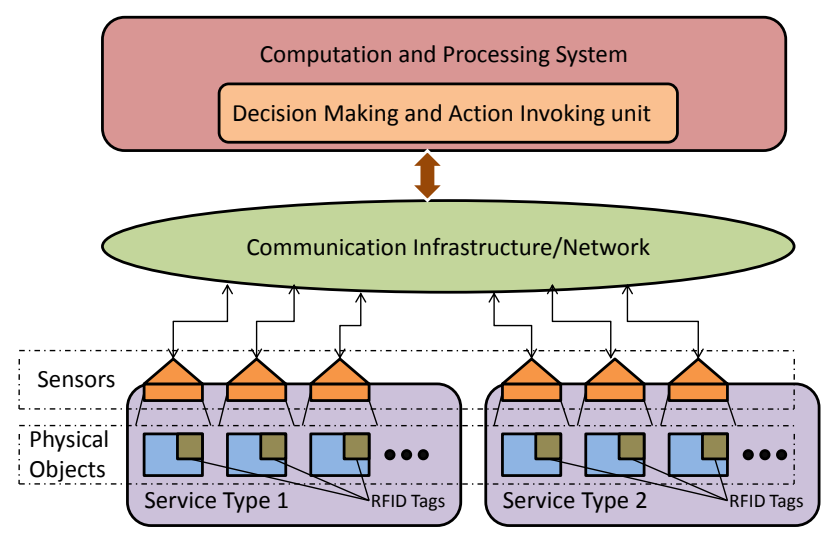

Figure 2: Basic IoT system.

proposed architecture for IoT needs to address many factors like scalability, interoperability, reliability, QoS, etc. Since IoT connects everything and everyone to exchange information among themselves, the traffic and storages in the network will also increase in the exponential way. Thus, IoT development depends on the technology progress and design of various new applications and business models. The basic architecture of IoT is proposed in [9] and [11].

Generally, the structure of IoT is divided into five layers as shown in Fig. 3. These layers are briefly described below:

1) Perception Layer: The Perception layer is also known as 'Device Layer'. It consists of the physical objects and sensor devices. The sensors can be RFID, 2D-barcode, or Infrared sensor depending upon objects identification method. This layer basically deals with the identification and collection of objects specific information by the sensor devices. Depending on the type of sensors, the information can be about location, temperature, orientation, motion, vibration, acceleration, humidity, chemical changes in the air etc. The collected information is then passed to Network layer for its secure transmission to the information processing system.

2) Network Layer: The Network layer can also be called 'Transmission Layer'. This layer securely transfers the information from sensor devices to the information processing system. The transmission medium can be wired or wireless and technology can be 3G, UMTS, Wifi, Bluetooth, infrared, ZigBee, etc depending upon the sensor devices. Thus, the Network layer transfers the information from Perception layer to Middleware layer.

3) Middleware Layer: The devices over the IoT implement different type of services. Each device connects and communicates with only those other devices which implement the same service type. This layer is responsible for the service management and has link to the database. It receives the information from Network layer and store in the database. It performs information processing and ubiquitous computation and takes automatic decision based on the results.

4) Application Layer: This layer provides global manage- 


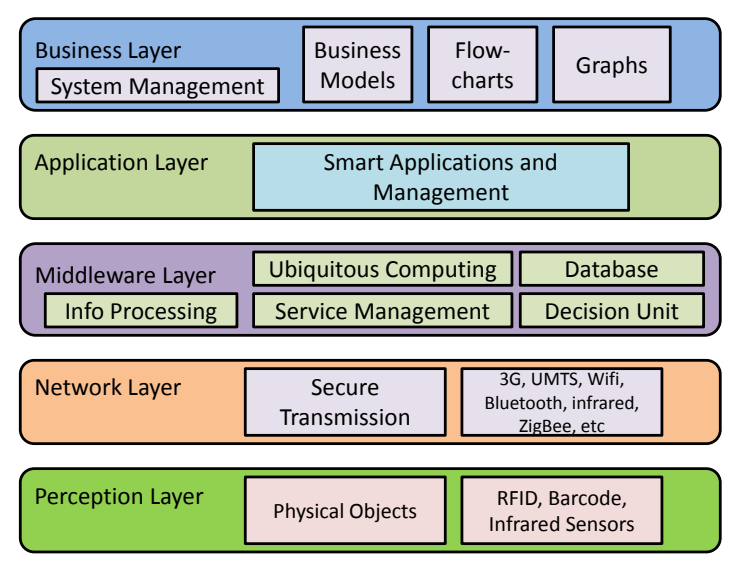

Figure 3: The IoT Architecture.

ment of the application based on the objects information processed in the Middleware layer. The applications implemented by IoT can be smart health, smart farming, smart home, smart city, intelligent transportation, etc.

5) Business Layer: This layer is responsible for the management of overall IoT system including the applications and services. It builds business models, graphs, flowcharts etc based on the data received from Application layer. The real success of the IoT technology also depends on the good business models. Based on the analysis of results, this layer will help to determine the future actions and business strategies.

\section{Possible Future Applications}

The IoT can find its applications in almost every aspect of our daily life. Below are some of the examples.

1) Prediction of natural disasters: The combination of sensors and their autonomous coordination and simulation will help to predict the occurrence of land-slides or other natural disasters and to take appropriate actions in advance.

2) Industry applications: The IoT can find applications in industry e.g., managing a fleet of cars for an organization. The IoT helps to monitor their environmental performance and process the data to determine and pick the one that need maintenance.

3) Water Scarcity monitoring: The IoT can help to detect the water scarcity at different places. The networks of sensors, tied together with the relevant simulation activities might not only monitor long term water interventions such as catchment area management, but may even be used to alert users of a stream, for instance, if an upstream event, such as the accidental release of sewage into the stream, might have dangerous implications.

4) Design of smart homes: The IoT can help in the design of smart homes e.g., energy consumption management, interaction with appliances, detecting emergencies, home safety and finding things easily, home security etc.

5) Medical applications: The IoT can also find applications in medical sector for saving lives or improving the quality of life e.g., monitoring health parameters, monitoring activities, support for independent living, monitoring medicines intake etc.

6) Agriculture application: A network of different sensors can sense data, perform data processing and inform the farmer through communication infrastructure e.g., mobile phone text message about the portion of land that need particular attention. This may include smart packaging of seeds, fertilizer and pest control mechanisms that respond to specific local conditions and indicate actions. Intelligent farming system will help agronomists to have better understanding of the plant growth models and to have efficient farming practices by having the knowledge of land conditions and climate variability. This will significantly increase the agricultural productivity by avoiding the inappropriate farming conditions.

7) Intelligent transport system design: The Intelligent transportation system will provide efficient transportation control and management using advanced technology of sensors, information and network. The intelligent transportation can have many interesting features such as non-stop electronic highway toll, mobile emergency command and scheduling, transportation law enforcement, vehicle rules violation monitoring, reducing environmental pollution, anti-theft system, avoiding traffic jams, reporting traffic incidents, smart beaconing, minimizing arrival delays etc

8) Design of smart cities: The IoT can help to design smart cities e.g., monitoring air quality, discovering emergency routes, efficient lighting up of the city, watering gardens etc.

9) Smart metering and monitoring: The IoT design for smart metering and monitoring will help to get accurate automated meter reading and issuance of invoice to the customers. The IoT can also be used to design such scheme for wind turbine maintenance and remote monitoring, gas, water as well as environmental metering and monitoring.

10) Smart Security: The IoT can also find applications in the field of security and surveillance e.g., surveillance of spaces, tracking of people and assets, infrastructure and equipment maintenance, alarming etc.

The IoT is also getting increasing popularity for academia, industry as well as government. Many international organizations are involved in the development of IoT. Microsoft's Eye-On-Earth platform creates an environment where water and air quality of a large number of European countries can be viewed, thus aiding in climate change research [8]. The European Commission is also involved in the research and development related to IoT. The Cluster of European Research Projects on the Internet of Things (CERP-IoT) is one of their active research project. The CERP-IoT look for IoT applications in societal, industrial and environmental domains [8]. The European FP7 project 'The Internet of Things Architecture' (IoT-A) focuses on the possible standard architecture for the IoT. Some other currently active European FP7 research projects that focus on the development of IoT includes IoT@Work, 'The Internet of Things Initiative' (IoTi) and 'European Research Cluster on the Internet of Things' (IERC). HP is also researching IoT based infrastructure in their 
Central Nervous System for the Earth initiative. Their aim is to populate the planet with billions of small sensors aimed at detecting vibrations and motion.

The IoT applications will continuously evolve with the passage of time but it has also to face many challenges related to privacy, security, scale and complexity, sufficient spectrum for connecting huge number of tagged objects or sensors etc. Some of the key challenges are addressed in section V.

\section{Key Challenges}

The IoT can change the shape of the Internet and can offer enormous economic benefits but it also faces many key challenges [10], [12]. Some of them are briefly described below.

1) Naming and Identity Management: The IoT will connect billions of objects to provide innovative services. Each object/sensor needs to have a unique identity over the Internet. Thus, an efficient naming and identity management system is required that can dynamically assign and manage unique identity for such a large number of objects.

2) Interoperability and Standardization: Many manufacturers provide devices using their own technologies and services that may not be accessible by others. The standardization of IoT is very important to provide better interoperability for all objects and sensor devices.

3) Information Privacy: The IoT uses different kind of object identification technologies e.g., RFID, 2D-barcodes etc. Since, every kind of daily use objects will carry these identification tags and embed the object specific information, it is necessary to take proper privacy measures and prevent unauthorized access.

4) Objects safety and security: The IoT consists of a very large number of perception objects that spread over some geographic area, it is necessary to prevent the intruder's access to the objects that may cause physical damage to them or may change their operation.

5) Data confidentiality and encryption: The sensor devices perform independent sensing or measurements and transfer data to the information processing unit over the transmission system. It is necessary that the sensor devices should have proper encryption mechanism to guarantee the data integrity at the information processing unit. The IoT service determines who can see the data, thus, it is necessary to guard the data from externals.

6) Network security: The data from sensor devices is sent over wired or wireless transmission network. The transmission system should be able to handle data from large number of sensor devices without causing any data loss due to network congestion, ensure proper security measures for the transmitted data and prevent it from external interference or monitoring.

7) Spectrum: The sensor devices will require dedicated spectrum to transmit data over the wireless medium. Due to limited spectrum availability, an efficient dynamic cognitive spectrum allocation mechanism is required to allow billions of sensors to communicate over the wireless medium.

8) Greening of IoT: The network energy consumption is increasing at very high rate due to increase in data rates, increase in the number of Internet-enabled services and rapid growth of Internet connected edge-devices. The future IoT will cause significant increase in the network energy consumption. Thus, green technologies need to be adopted to make the network devices as energy efficient as possible.

\section{CONCLUSIONS}

This paper introduced the emerging future form of Internet called "Internet of Things" that will connect everything and everyone. The IoT embeds intelligence in the sensor devices to autonomously communicate, exchange information and take intelligent decisions. Simply, IoT transitions human-human communication to human-human, human-device and devicedevice communication. This paper described briefly the evaluation of Internet, proposed the generic structure for IoT, described possible future applications and some active international projects in the field of IoT and finally addressed some key challenges associated with the IoT technology. The IoT deployment could be hard and require large research efforts to tackle with the challenges but it can provide significant personal, professional and economic benefits in the near future.

\section{REFERENCES}

[1] J. Zheng, D. Simplot-Ryl, C. Bisdikian, and H. Mouftah, "The Internet of Things," in IEEE Communications Magazine, Volume:49, Issue: 11, pp:30-31, 2011.

[2] Y. Huang and G. Li, "Descriptive Models for Internet of Things," in IEEE International Conference on Intelligent Control and Information Processing (ICICIP), August 2010.

[3] T. Fan and Y. Chen, "A Scheme of Data Management in the Internet of Things," in 2nd IEEE International Conference on Network Infrastructure and Digital Content, Sept. 2010.

[4] Y. Huang and G. Li, "A Semantic Analysis for Internet of Things," in International Conference on Intelligent Computation Technology and Automation (ICICTA), May 2010.

[5] Q. Zhou and J. Zhang, "Research Prospect of Internet of Things Geography," in 19th International Conference on Geoinformatics, June 2011.

[6] J. Li, Z. Huang, and X. Wang, "Countermeasure Research about Developing Internet of Things Economy," in International Conference on E -Business and E-Government (ICEE), May 2011.

[7] Y. Yu, J. Wang, and G. Zhou, "The Exploration in the Education of Professionals in Applied Internet of Things Engineering," in 4th International Conference on Distance Learning and Education (ICDLE), October 2010.

[8] L. Coetzee and J. Eksteen, "The Internet of Things: Promise for the Future? An Introduction," in IST-Africa Conference Proceedings, CSIR, Pretoria, South Africa, May 2011.

[9] L. Tan and N. Wang, "Future Internet: The Internet of Things," in 3rd International Conference on Advanced Computer Theory and Engineering (ICACTE), August 2010.

[10] G. Gang, L. Zeyong, and J. Jun, "Internet of Things Security Analysis," in International Conference on Internet Technology and Applications (iTAP), August 2011.

[11] M. Wu, T. Lu, F. Ling, J. Sun, and H. Du, "Research on the Architecture of Internet of Things," in 3rd International Conference on Advanced Computer Theory and Engineering (ICACTE), Aug. 2010.

[12] Z. Hu, "The research of several key question of Internet of Things," in International Conference on Intelligence Science and Information Engineering (ISIE), August 2011. 